\title{
Review of Asian Options
}

\author{
Jiaying Han, Yicheng Hong* \\ College of Science, Yanbian University, Yanji, China \\ Email: *kayieng@126.com
}

How to cite this paper: Han, J.Y. and Hong, Y.C. (2022) Review of Asian Options. Open Access Library Journal, 9: e8358. https://doi.org/10.4236/oalib.1108358

Received: January 10, 2022

Accepted: February 12, 2022

Published: February 15, 2022

Copyright (C) 2022 by author(s) and Open Access Library Inc.

This work is licensed under the Creative

Commons Attribution International

License (CC BY 4.0).

http://creativecommons.org/licenses/by/4.0/

\begin{abstract}
Option, is a right that gives the purchaser a contract to buy or sell a certain underlying asset at a certain price at a certain time in the future. Asian options are one of the representative products, and it is also the most active new type of option in the financial derivatives market today. However, the path dependence characteristics make the pricing model of Asian options show a relatively large difference compared with that of standard options, and the pricing problem is far more complicated than that of European options. In order to facilitate the research of more scholars, this paper summarizes the Asian Options. The first part of this paper introduces the definition and classifications of Asian options. The second part introduces several feasible methods for Asian option pricing in detail. In the third part, it is found that the Monte Carlo simulation method is a good approximation to the arithmetic average Asian option.
\end{abstract}

\section{Subject Areas}

Financial Mathematics

\section{Keywords}

Asian Options, Option Pricing, Monte Carlo Simulation, Geometric Average

\section{Introduction}

Asian option, also known as average option, is a derivative of stock option and one of the most active options in today's financial market. In the 1980s, the banking industry in Europe and America was in a period of change. In 1984, the Bank of England passed the bill on foreign exchange option trading in British banking industry. Mark Standish, a trader at Bankers Trust in London, has been engaged in fixed income derivatives and related arbitrage transactions. David *Corresponding author. 
Spaughton has been engaged in system analysis at trust bank since 1984. In 1987, Standish and Spaghton successfully cooperated in Tokyo, Japan to develop option formula for pricing crude oil average price option which can be widely used in the financial industry. They name this kind of exotic options as Asian options. Its essence is the innovation of European options. The same point with European options is that they only allow investors to execute the option contract on the expiration date. The difference is that the income of European options depends on the price of the underlying asset on the expiration date, and the income of Asian options depends on the historical average price of the underlying asset. Asian options are popular in the option market because they adopt an average value and reduce volatility, making them cheaper than other standard options [1]. However, its price has no analytical expression. So far, the Asian options pricing is still an open problem. It is assumed that the underlying asset price obeys the lognormal distribution, the geometric mean of a series of lognormal distribution variables still obeys the lognormal distribution [2] [3], and the corresponding arithmetic mean has no analytical solution. Therefore, exploring its reasonable value estimation method has become a topic of important academic value in option theory.

\section{Classifications of Asian Options}

To accurately define the average value used in Asian option contracts depends on some factors: how the data points are combined into the average value and which data points are used. The former refers to whether to use arithmetic average, geometric average or other more complex averages. The latter refers to how many data points to use when calculating the average, whether to use all quotations or just a subset, and for which period.

\subsection{Arithmetic or Geometric}

The two simplest and most common types of averages are arithmetic average and geometric average. The arithmetic mean of prices is the sum of all prices with equal weights, divided by the total number of prices used, and then indexed. Another common calculation is index weighted average, which means that the new price is given a higher weight than the old price in the way of exponential decline instead of equal weight before calculating the average price.

\subsection{Discrete or Continuous}

Depend on how much data used to calculate the average, and whether use the full transaction price or only a subset, Asian options can be divided into different types. If the prices closely arranged in a limited period of time are used, the sum formula used in the calculation of the average value is the integral of the asset price (or function) in that period of time. The result is a continuously sampled average. More often, we only use some reliable data points, such as a smaller data set like closing price. This is called discrete sampling. 


$$
\text { Option Pricing } \begin{cases}\text { Discrete } \begin{cases}\text { Geometric Average } & J_{n}=\frac{1}{n+1} \sum_{0}^{1} S_{t_{i}} \\ \text { Arithmetic Average } & J_{n}=\mathrm{e}^{\frac{1}{n+1}} \sum_{0}^{1} S_{t_{i}}\end{cases} \\ \text { Continuosly } \begin{cases}\text { Geometric Average } & J_{t}=\frac{1}{t} \int_{0}^{t} S_{\tau} \mathrm{d} \tau \\ \text { Arithmetic Average } & J_{t}=\mathrm{e}^{\frac{1}{t}} \int_{0}^{t} S_{\tau} \mathrm{d} \tau\end{cases} \end{cases}
$$

\subsection{Standard or Forward}

For discrete cases, according to the initial price and average price of the underlying asset, discrete Asian options can be divided into two types. If the initial price is included in the average price, it is called Standard Asian option. If the initial price is not included in the average price, it is called Forward-starting Asian option.

$$
A_{i}= \begin{cases}\frac{1}{i+1} \sum_{j=0}^{i} S_{j} & \text { Standard Asian Options } \\ \frac{1}{i} \sum_{j=1}^{i} S_{j} & \text { Forward Sarting Asian Options }\end{cases}
$$

\subsection{Fixed or Floating}

According to the return at maturity, Asian options can be divided into following two types. If the return depends on the difference between the historical average price of the underlying asset and a fixed price, it is called Fixed Strike Asian Option. If the return depends on the difference between the historical average price of the underlying asset and the price on the maturity date, it is called Floating Strike Asian Option.

Returns on Asian options with fixed strike price

$$
= \begin{cases}\max \left(J_{T}-K, 0\right) & \text { Call Option } \\ \max \left(K-J_{T}, 0\right) & \text { Put Option }\end{cases}
$$

Returns on Asian options with floating strike price

$$
= \begin{cases}\max \left(S_{T}-S_{T}, 0\right) & \text { Call Option } \\ \max \left(J_{T}-S_{T}, 0\right) & \text { Put Option }\end{cases}
$$

where, $J$ represents the historical average price of the underlying asset, $K$ represents the fixed price (called the execution price) of the underlying asset, and $S$ represents the price on the maturity date of the underlying asset.

\section{Asian Option Pricing Method}

\subsection{Geometric Average Asian Options Pricing}

Let the price $S$ of the underlying asset satisfies the following stochastic differential equation:

$$
\mathrm{d} S=r S \mathrm{~d} t+\sigma S \mathrm{~d} W(t)
$$

where, $t \geq 0$ represents time, $r>0$ represents risk-free interest rate, $\sigma>0$ 
represents drift rate, $W(t), t \geq 0$ represents the Wiener process. Then,

$$
S(t)=S(0) \exp \left\{\left(r-\frac{1}{2} \sigma^{2}\right) t+\sigma W(t)\right\}
$$

In risk neutral pricing, the validity period $[0, T]$ of the option is divided into $\left[t_{0}, t_{1}\right],\left[t_{1}, t_{2}\right], \cdots,\left[t_{n-1}, t_{n}\right] . S_{1}, S_{2}, \cdots, S_{n}$ are the values of the stock price at different times $t_{1}, t_{2}, \cdots, t_{n}$.

Geometric average value of stock is

$$
A^{G}=\left(\prod_{i=1}^{n} S_{i}\right)^{\frac{1}{n}}=\exp \left\{\frac{\sum_{i=1}^{n} \ln S_{i}}{n}\right\}
$$

let $Y=\frac{\sum_{i=1}^{n} \ln S_{i}}{n}$, then

$$
\ln S_{n}=\ln S_{0}+\left(r-\frac{\sigma^{2}}{2}\right) n t+\sigma \varepsilon \sqrt{n t}
$$

where, $\varepsilon$ is a random variable subject to standard normal distribution.

Therefore,

$$
\ln S_{i} \sim N\left(\ln S_{0}+\left(r-\frac{\sigma^{2}}{2}\right) i t, \sigma^{2} i t\right)
$$

According to the properties of the normal distribution, $Y$ also obeys the normal distribution, and $\ln S_{i}$ obeys the normal distribution with the mean of $\left(r-\frac{\sigma^{2}}{2}\right) t$ and the variance of $\sigma^{2} t$. We can get

$$
\begin{aligned}
E[Y] & =E\left[\frac{\sum_{i=1}^{n} \ln S_{i}}{n}\right] \\
& =E\left[\frac{\ln S_{1}+\ln S_{2}+\cdots+\ln S_{n}}{n}\right] \\
\operatorname{Var}[Y] & =\frac{1}{n^{2}} \operatorname{Var}\left[\ln S_{1}+\ln S_{2}+\cdots+\ln S_{n}\right] \\
& \left.=\frac{\sigma^{2}}{2}\right) t \\
& =\frac{1}{n^{2}} \operatorname{Var}\left[\operatorname { l n } \left(S_{0}+\frac{n+1}{2 n}\left(r-\frac{\sigma^{2}}{2}\right) T\right.\right. \\
& =\frac{1}{n^{2}} \operatorname{Var}\left[\ln \frac{S_{n} S_{n-1}^{2} S_{n-2}^{3} \cdots S_{1}^{n}}{S_{n-1} S_{n-2}^{2} S_{n-3}^{3} \cdots S_{0}^{n}} S_{0}^{n}\right] \\
& =\frac{1}{n^{2}}\left(\sigma^{2} t+2^{2} \sigma^{2} t+3^{2} \sigma^{2} t+\cdots+n^{2} \sigma^{2} t\right) \\
& =\frac{(n+1)(2 n+1)}{6 n^{2}} \sigma^{2} T \\
& \\
& \\
&
\end{aligned}
$$


Therefore,

$$
\begin{gathered}
Y=\ln A^{G} \sim N\left(\ln S_{0}+\frac{n+1}{2 n}\left(r-\frac{\sigma^{2}}{2}\right) T, \frac{(n+1)(2 n+1)}{6 n^{2}} \sigma^{2} T\right) \\
\ln \frac{A^{G}}{\ln S_{0}} \sim N\left(\frac{n+1}{2 n}\left(r-\frac{\sigma^{2}}{2}\right) T, \frac{(n+1)(2 n+1)}{6 n^{2}} \sigma^{2} T\right)
\end{gathered}
$$

let $\left(\mu_{G}-\frac{\sigma_{G}^{2}}{2}\right) T=\frac{n+1}{2 n}\left(r-\frac{\sigma^{2}}{2}\right) T, \sigma_{G}^{2} T=\frac{(n+1)(2 n+1)}{6 n^{2}} \sigma^{2} T$, then

$$
\begin{gathered}
\sigma_{G}^{2}=\frac{(n+1)(2 n+1)}{6 n^{2}} \sigma^{2} \\
\mu_{G}=\frac{n+1}{2 n}\left(r-\frac{\sigma^{2}}{2}\right)+\frac{\sigma_{G}^{2}}{2}
\end{gathered}
$$

Because $A^{G}$ obeys lognormal distribution,

$$
E\left[A^{G}\right]=\exp \left\{\left[\ln S_{0}+\left(\mu_{G}-\frac{\sigma_{G}^{2}}{2}\right) T\right]+\frac{\sigma_{G}^{2}}{2} T\right\}=S_{0} \mathrm{e}^{\mu_{G} T},
$$

the price of discrete geometric average Asian option call option is

$$
\begin{aligned}
& \qquad \begin{aligned}
C^{G}\left(S_{0}, K, T\right) & =\mathrm{e}^{-r T} E\left[A^{G}-K\right]^{+} \\
& =\mathrm{e}^{-r T}\left\{E\left[A^{G}\right] N\left(d_{1}\right)-K N\left(d_{2}\right)\right\} \\
& =\mathrm{e}^{-r T}\left\{S_{0} \mathrm{e}^{\mu_{G} T} N\left(d_{1}\right)-K N\left(d_{2}\right)\right\} \\
& =S_{0} \mathrm{e}^{\left(\mu_{G}-r\right) T} N\left(d_{1}\right)-K \mathrm{e}^{-r T} N\left(d_{2}\right)
\end{aligned} \\
& \text { where } d_{1}=\frac{\ln \frac{S_{0}}{K}+\left(\mu_{G}+\frac{\sigma_{G}^{2}}{2}\right) T}{\sigma_{G} \sqrt{T}}, d_{2}=d_{1}-\sigma_{G} \sqrt{T} .
\end{aligned}
$$

\subsection{Monte Carlo Simulation Option Pricing}

Monte Carlo simulation is a numerical method to solve approximate solutions of mathematical, physical and engineering problems by sampling relevant random variables [3]. It is also the basis of computer simulation. In recent years, it has been widely used in the financial field and achieved remarkable results. The basic idea of Monte Carlo is to construct a random variable so that let a certain numerical characteristic be the solution of the problem, then sample the random variable, calculate the parameter value corresponding to each sample which is the random solution of the problem, and finally make statistical analysis on each random solution obtained in the sampling process [4]. Therefore, the probability solution of the problem and the accuracy estimation of the solution are calculated. It is expected that the accuracy will gradually increase with the increase of sampling times. On the premise of improving the accuracy, a large number of 
repeated calculations are generally required. The following is a brief introduction to Monte Carlo simulation of option pricing and controlled variable Monte Carlo simulation [5] [6] [7] [8] [9].

In the risk neutral market, the price of European call option at time 0 is $v=\mathrm{e}^{-r \tau} E\left(S_{\tau}-K\right)^{+}$, let $Y=\left(S_{\tau}-K\right)^{+}$, assuming that $Y_{1}, Y_{2}, \cdots, Y_{n}$ is a set of independent and identically distributed samples of $Y, V^{j}=\mathrm{e}^{-r \tau} Y_{j}, V_{1}, V_{2}, \cdots, V_{n}$ is a group of independent and identically distributed samples of $V$. Then $\bar{V}=\frac{1}{n} \sum_{j=1}^{n} V_{j}$ can be seen as an unbiased estimation of $V$. According to the strong law of large numbers, there are

$$
\bar{V}=\frac{1}{n} \sum_{i=1}^{n} V_{i} \rightarrow v
$$

$\bar{V}$ is the estimation formula of Monte Carlo simulation, and its standard deviation is as follow:

$$
\sigma_{\bar{V}}=\sqrt{\operatorname{Var} \bar{V}}=\frac{1}{\sqrt{n}} \sqrt{\operatorname{Var} V}=\frac{1}{\sqrt{n}} \sigma
$$

In order to improve the simulation accuracy, a large number of scholars have proposed variance reduction techniques, such as control variable method, dual variable method and important variable method. Among them, the control variable method is the most common and widely used method in option pricing [7].

A variable $Y^{*}$ related to $Y$ is introduced, where $v^{*}=\mathrm{e}^{-r \tau} E Y^{*}$. Let $V^{*}=\mathrm{e}^{-r \tau} Y^{*}$, and $v^{*}$ is the control variable. Assume $\left(Y_{1}, Y_{1}^{*}\right),\left(Y_{2}, Y_{2}^{*}\right), \cdots$, $\left(Y_{n}, Y_{n}^{*}\right)$ are a group of independent and identically distributed samples of $\left(Y, Y^{*}\right)$, and $\left(V_{1}, V_{1}^{*}\right),\left(V_{2}, V_{2}^{*}\right), \cdots,\left(V_{n}, V_{n}^{*}\right)$ are a group of independent and identically distributed samples of $\left(V, V^{*}\right)$.

Note that $\bar{V}=\frac{1}{n} \sum_{i=1}^{n} V_{i}, \overline{V^{*}}=\frac{1}{n} \sum_{j=1}^{n} \overline{V_{i}^{*}}$. Let $\bar{V}_{c}=\bar{V}+c\left(v^{*}-\overline{V^{*}}\right)$, where, $C$ is a constant and $\bar{V}_{c}$ is the unbiased estimation of $v$. Therefore, $\bar{V}_{c}$ is the estimation formula of option price $V$ in Monte Carlo simulation of control variable, and the variance is as follow:

$$
\operatorname{Var}\left(\bar{V}_{c}\right)=\operatorname{Var}(\bar{V})+c^{2} \operatorname{Var}\left(\overline{V^{*}}\right)-2 c \operatorname{Cov}\left(V, \overline{V^{*}}\right)
$$

If select the value of $C$ properly, $\operatorname{Var}\left(\bar{V}_{c}\right)$ can be minimized, because

$$
\begin{gathered}
\frac{\mathrm{d}}{\mathrm{d} c} \operatorname{Var}\left(\bar{V}_{c}\right)=2 c \operatorname{Var}\left(V^{*}\right)-2 c \operatorname{Cov}\left(V, \overline{V^{*}}\right) \\
\frac{\mathrm{d}^{2}}{\mathrm{~d} c^{2}}=2 \operatorname{Var}\left(\overline{V^{*}}\right) \geq 0
\end{gathered}
$$

Therefore, $c^{*}=\frac{\operatorname{Cov}\left(V, \overline{V^{*}}\right)}{\operatorname{Var}\left(\overline{V^{*}}\right)}$ makes $\operatorname{Var}\left(\bar{V}_{c}\right)$ minimum, the minimum value is 


$$
\begin{aligned}
\operatorname{Var}\left(\bar{V}_{c}\right) & =\operatorname{Var}(\bar{V})-\frac{\operatorname{Cov}\left(V, \overline{V^{*}}\right)}{\operatorname{Var}\left(V^{*}\right)} \\
& =\left[1-\frac{\operatorname{Cov}\left(V, \overline{V^{*}}\right)}{\operatorname{Var}\left(V^{*}\right)} \operatorname{Var}(\bar{V})\right] \operatorname{Var}(\bar{V}) \\
& =\left[1-\operatorname{Corr}^{2}\left(V, \overline{V^{*}}\right)\right] \operatorname{Var}(\bar{V})
\end{aligned}
$$

With $\rho=\operatorname{Corr}\left(Y, Y^{*}\right)=\operatorname{Corr}\left(V, V^{*}\right)$, we can get

$$
\operatorname{Var}\left(\bar{V}_{c}\right)=\left(1-\rho^{2}\right) \operatorname{Var}(\bar{V})
$$

It can be seen that the variance of control variable Monte Carlo simulation is less than that of Monte Carlo simulation, and when the correlation coefficient between control variable and original variable is large, the variance of control variable Monte Carlo simulation is much smaller than that of Monte Carlo simulation. The value of arithmetic mean Asian options at time 0 depends on arithmetic mean of the underlying asset simulation path $S_{i}(i=1,2, \cdots, \tau)$, which is $\frac{1}{\tau} \sum_{i=1}^{\tau} S_{i}$. The correlation coefficient between the control variable and the option to be simulated is large. Let the form of sum of multiple variables $V^{*}=\frac{1}{\tau} \sum_{i=1}^{\tau} S_{i}$ as the control variable, so that $\bar{V}_{c}=\bar{V}+c\left(v^{*}-\overline{V^{*}}\right)$, where $\overline{V^{*}}=\frac{1}{\tau} \sum_{i=1}^{\tau} V_{i}$, the stock price $S$ follows geometric Brownian motion, distribution of $\sum_{i=1}^{\tau} S_{i}$ is uncertain. Therefore, the approximate distribution should be considered when calculating the correlation coefficient between $V^{*}$ and $V$.

$$
\begin{aligned}
\rho & =\operatorname{Corr}\left(V^{*}, V\right)=\frac{E\left[\left(V^{*}-E \overline{V^{*}}\right)(V-E V)\right]}{\sqrt{\operatorname{Var}\left(V^{*}\right) \operatorname{Var}(V)}} \\
& =\frac{E\left[\left(\frac{1}{\tau} \sum_{i=1}^{\tau} S_{i}-K\right)\left(\sum_{i=1}^{\tau} S_{i}\right)\right]+E\left(\frac{1}{\tau} \sum_{i=1}^{\tau} S_{i}-K\right) E\left(\sum_{i=1}^{\tau} S_{i}\right)}{\sqrt{\operatorname{Var}\left(\frac{1}{\tau} \sum_{i=1}^{\tau} S_{i} / \tau-K\right)} \operatorname{Var}\left(\sum_{i=1}^{\tau} S_{i}\right)}
\end{aligned}
$$

Since the distribution of $A_{\tau}=\sum_{i=1}^{\tau} \frac{S_{i}}{\tau}$ can not be determined, so the geometric Brownian motion $Q_{\tau}=S_{0} \mathrm{e}^{X_{\tau}}$ is used. Among them,

$$
\begin{aligned}
& X_{\tau} \sim N\left(\frac{\bar{\mu}-\bar{\sigma}^{2}}{2} \tau, \bar{\sigma}^{2} \tau\right) \\
& \bar{\mu}=\frac{1}{\tau} \ln \frac{\mathrm{e}^{r}\left(1-\mathrm{e}^{\tau r}\right)}{\tau\left(1-\mathrm{e}^{-r}\right)} \\
& \bar{\sigma}=\frac{-4 \bar{\mu}+2 \ln \left[E\left(A_{\tau}\right) / S_{0}^{2}\right]}{\tau\left(1-\mathrm{e}^{r}\right)}
\end{aligned}
$$




\section{Summary}

This paper introduces the Asian Options and option pricing methods. The details of the related models are sorted out. The Geometric average model can get the exact analytical solution though, in the real financial market, most Asian options are usually arithmetic average Asian options. Due to the arithmetic mean of lognormal random variables is no longer lognormal distribution, it is difficult to find the exact analytical solution of arithmetic mean Asian option. Therefore, many scholars and practical financial workers have proposed effective numerical simulation pricing algorithms for arithmetic average Asian options based on various models, such as Monte Carlo simulations method. This paper summarizes the research theory of Asian option pricing, and proposes to use Monte Carlo simulation to price arithmetic average Asian option, which plays a certain reference role for the academic research on Asian option pricing.

\section{Conflicts of Interest}

The authors declare no conflicts of interest regarding the publication of this paper.

\section{References}

[1] Horvath, A. and Medvegyev, P. (2016) Pricing Asian Options: A Comparison of Numerical and Simulation Approaches Twenty Years Later. Journal of Mathematical Finance, 6, 810-841. https://doi.org/10.4236/jmf.2016.65056

[2] Turnbull, S. and Wakeman, L. (1991) A Quick Algorithm for Pricing European Average Options. Journal of Financial and Quantitative Analysis, 26, 309-326. https://doi.org/10.2307/2331213

[3] Levy, E. (1992) The Valuation of Average Rate Currency Options. Journal of International Money and Finance, 11, 474-491. https://doi.org/10.1016/0261-5606(92)90013-N

[4] Boyle, P.P. (1977) Options: A Monte Carlo Approach. Journal of Financial Economics, 4, 323-338. https://doi.org/10.1016/0304-405X(77)90005-8

[5] Boyle, P.P. and Emanuel, D. (1980) Discretely Adjusted Option Hedges. Journal of Financial Economics, 8, 259-282. https://doi.org/10.1016/0304-405X(80)90003-3

[6] Dingec, K.D. and Hormann, W. (2013) Control Variates and Conditional Monte Carlo for Basket and Asian Options. Journal of Insurance Mathematics and Economics, 3, 421-434. https://doi.org/10.1016/j.insmatheco.2013.03.002

[7] Kemma, A.G. and Vorst, A.C. (1900) A Pricing Method for Options Based on Awerge Asset Values. Journal of Banking and Finance, 4, 121-168.

[8] Caflisch, R. (1998) Monte Carlo and Quasi-Monte Carlo Methods. Acta Numerica, 6, 1-49. https://doi.org/10.1017/S0962492900002804

[9] Cox, J., Ross, S. and Rubinstein, M. (1979) Option Pricing: A Simplified Approach. Journal of Financial Economics, 7, 229-264.

https://doi.org/10.1016/0304-405X(79)90015-1 